\title{
Prediction of solar activity on the basis of spectral characteristics of sunspot number
}

\author{
E. Echer ${ }^{1}$, N. R. Rigozo ${ }^{1,2}$, D. J. R. Nordemann ${ }^{1}$, and L. E. A. Vieira ${ }^{1}$ \\ ${ }^{1}$ Instituto Nacional de Pesquisas Espaciais (INPE), Av. Astronautas, 1758 ZIP 12201-970, São José dos Campos, SP, Brazil \\ ${ }^{2}$ Faculdade de Tecnologia Thereza Porto Marques (FAETEC) ZIP 12308-320, Jacareí, Brazil
}

Received: 9 July 2003 - Revised: 6 February 2004 - Accepted: 18 February 2004 - Published: 14 June 2004

\begin{abstract}
Prediction of solar activity strength for solar cycles 23 and 24 is performed on the basis of extrapolation of sunspot number spectral components. Sunspot number data during 1933-1996 periods (solar cycles 17-22) are searched for periodicities by iterative regression. The periods significant at the $95 \%$ confidence level were used in a sum of sine series to reconstruct sunspot series, to predict the strength of solar cycles 23 and 24. The maximum peak of solar cycles is adequately predicted (cycle 21: $158 \pm 13.2$ against an observed peak of 155.4 ; cycle 22 : $178 \pm 13.2$ against 157.6 observed). Solar cycle 23 was predicted to have a peak in 2000 with maximum amplitude of $125 \pm 13.2$, in good agreement with the 119.6 observed. The minimum of solar cycle 23 is predicted to occur around 2007-2008. For solar cycle 24 , the maximum is predicted to occur in $2012(115 \pm 13.2)$ or $2013(117 \pm 13.2)$ and this shall be a very weak solar cycle.
\end{abstract}

Key words. Solar physics, astrophysics and astronomy (instruments and techniques; general or miscellaneous; photosphere and chromosphere)

\section{Introduction}

The most dramatic, easily observable and long-term recorded aspect of solar activity are the dark regions on the solar disk - sunspots, which have been observed telescopically since 1610. Their number per time interval may, in one way or another, represent an index of the general solar magnetic activity (Eddy, 1976, 1977; Hoyt and Schatten, 1997, 1998a, b). The number of sunspots shows time variations in a distinctly cyclic fashion. Quantitatively, they are measured by the Wolf (or Zurich) Sunspot Number, $\mathrm{R}_{z}$, which is widely used in solar and solar-terrestrial physics, as a proxy for the general state of solar activity. $\mathrm{R}_{z}$ is more reliable after 1850 , when daily averages are more frequently available (Hoyt and Schatten, 1998a, b).

When the solar cycle is in its maximum phase, there are important terrestrial consequences, such as the higher solar emission of extreme-ultraviolet and ultraviolet flux, which can modulate the middle and upper terrestrial atmosphere, and total solar irradiance, which could have effects on terrestrial climate (Hoyt and Schatten, 1997), as well as the coronal mass ejection and interplanetary shock rates, responsible by geomagnetic activity storms and auroras (Webb and Howard, 1994). There is also a high probability for the occurrence of large solar flares, with associated energetic solar particles causing phenomena such as communications disturbances, failures in electronic solid state components, etc. (Siscoe, 2000). The strength of these events depends on the size of solar cycle activity, which is extremely variable. These facts justify the scientific and practical importance of predicting the solar cycle strength.

Ideally, one should be able to predict future solar activity using a realistic model of the solar dynamo, but this model does not yet exist. Two approaches are generally used to predict solar activity (after Layden et al., 1991; see also Conway, 1998):

1. Purely numeric methods. These methods are based on the search of multiple periodicities and amplitudes able to reproduce past solar cycles. Their main advantage is that any future solar cycle can be predicted, independent of how far in the future it occurs. The drawback is that no physical information is used. The prediction supposes that an important part of the phenomena is really periodic and that most important periodicities have been already observed in data. Another advantage is that this method can also be used, with the same restriction, to reconstruct past solar activity before the measurements were recorded (e.g. Rigozo et al., 2001; Echer et al., 2004). 
Table 1. Results of solar cycle 23 peak prediction by several authors using different techniques.

\begin{tabular}{llll}
\hline Prediction & Max \#23 & Prediction & Max \#23 \\
\hline Schatten and Pesnell (1993) & $170 \pm 25$ & Schatten et al. (1996) & $138 \pm 30$ \\
Schatten and Sofia (1996) & $130 \pm 30$ & Thompson (1996) & 164 \\
Bounar et al. (1997) & $158 \pm 18$ & Jain (1997) & 166.2 \\
Kane (1997) & $170 \pm 13$ & Li (1997) & $149.3 \pm 20$ \\
Tsirulnik et al. (1997) & 220 & Hathaway et al. (1999) & $146 \pm 20$ \\
Kane (1999) & $140 \pm 9$ & Mendoza and Ramírez (1999) & $140 \pm 32$ \\
Ahluwalla (2000) & $131.5 \pm 33$ & Ramesh (2000) & $126 \pm 26$ \\
Loskutov et al. (2001) & 122 & This work & $125 \pm 13.2$ \\
\hline Observed & 119.6 & & \\
\hline
\end{tabular}

2. Precursor techniques: These techniques are statistical in nature and are usually based on correlating any solar/geophysical parameter from earlier in the solar cycle to sunspot numbers at solar maximum or some other point later in the cycle. Precursor methods are considered to be the more successful techniques and have been widely employed (Ohl, 1966; Kane, 1978; Layden et al., 1991), although they have failed in predicting the solar cycle 23 maximum (Kane, 2001; see Table 1 in this paper). In the present work, an iterative regression is applied in sunspot number annual averages, in order to obtain the most significant periodicities, which are used in a reconstruction and a prediction of solar activity for cycles 23 and 24 .

\section{Sunspot data and spectral analysis methods}

\subsection{Sunspot data}

The annual averages of the sunspot number were used in this work. The sunspot number time series was obtained from the Sunspot Index Data Center in Brussels, Belgium.

\subsection{Spectral analysis}

The iterative regression method is an iterative least-square fit and it was described in its general form by Wolberg (1967) and Rigozo and Nordemann (1998). It may be applied to the fit of any function to experimental or observational data, without need of previous linearization. To keep processing times to a minimum, the number of parameters and variables cannot be too high but there is no such practical limit for the number of data points. It is applied in this work to searching for periodicities in a time series, with the use, for every period searched, of a unique sine function with three unknown parameters, $\mathrm{a}_{0}=$ amplitude, $\mathrm{a}_{1}=$ angular frequency, and $a_{2}=$ phase and only one variable, $t=t$ time. The values for the three unknown parameters are determined one at a time by iteration on the original time series with the specific condition of restricting the angular frequency to a narrow domain around its expected value. The starting point of the method is the definition of a so-called conditional function:

$F=Y-a_{0} \sin \left(a_{1} t+a_{2}\right)$,

where $Y$ is the observed signal/value. Clearly $F$ is the difference between the observed value and the fitted function.

This method has the ability to detect a periodic signal even if only part of the full cycle is sampled. It also gives estimatives of the error for every frequency, amplitude and phase calculated. Based on these advantages, it was selected and applied to the sunspot number annual averages and the most significant periods at $2 \sigma$ were chosen for the reconstruction of the sunspot number. A sum of sine waves is calculated, using the parameters' fit by the ARIST method, according to Eq. (2):

$f(t)=\sum_{1}^{n} r_{n} \sin \left(w_{n} t+\phi_{n}\right)$.

More details regarding the ARIST method can be found in Rigozo and Nordemann (1998). A comparison among ARIST and other spectral methods will be published elsewhere.

\section{Results and discussion}

Since the Wolf Sunspot number is more reliable after 1850 (Hoyt and Schatten, 1998a, b), only sunspot data after this date are used in this work. Long periods could be important in describing the future solar activity (Kane, 2002). However, it was observed in several spectral analyses that changes in the spectrum can occur due to the sample selection (Herman and Goldberg, 1978; Kane, 1999). Considering these two points, several sample lengths were tested in order to choose the ones that best predicted the sunspot series in the reconstruction and that gave the most accurate sunspot maximum values.

Figure 1 shows the sunspot series during 1850-2000. The amplitude of sunspot cycles was lower during the 19th century than the 20th, especially after 1940 . This strong difference in amplitude could influence a prediction of near future 


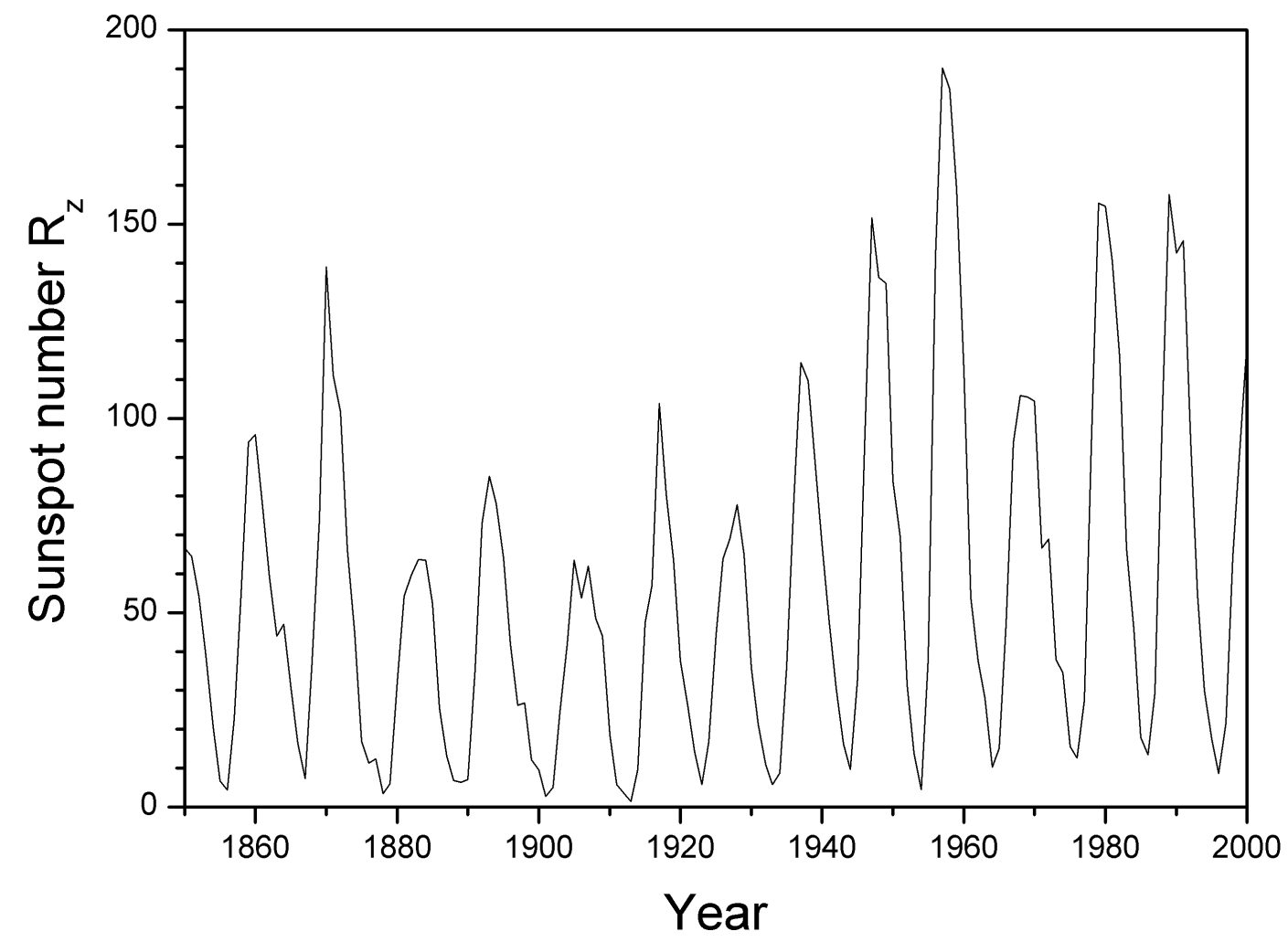

Fig. 1. Sunspot number time series in the period 1850-2000.

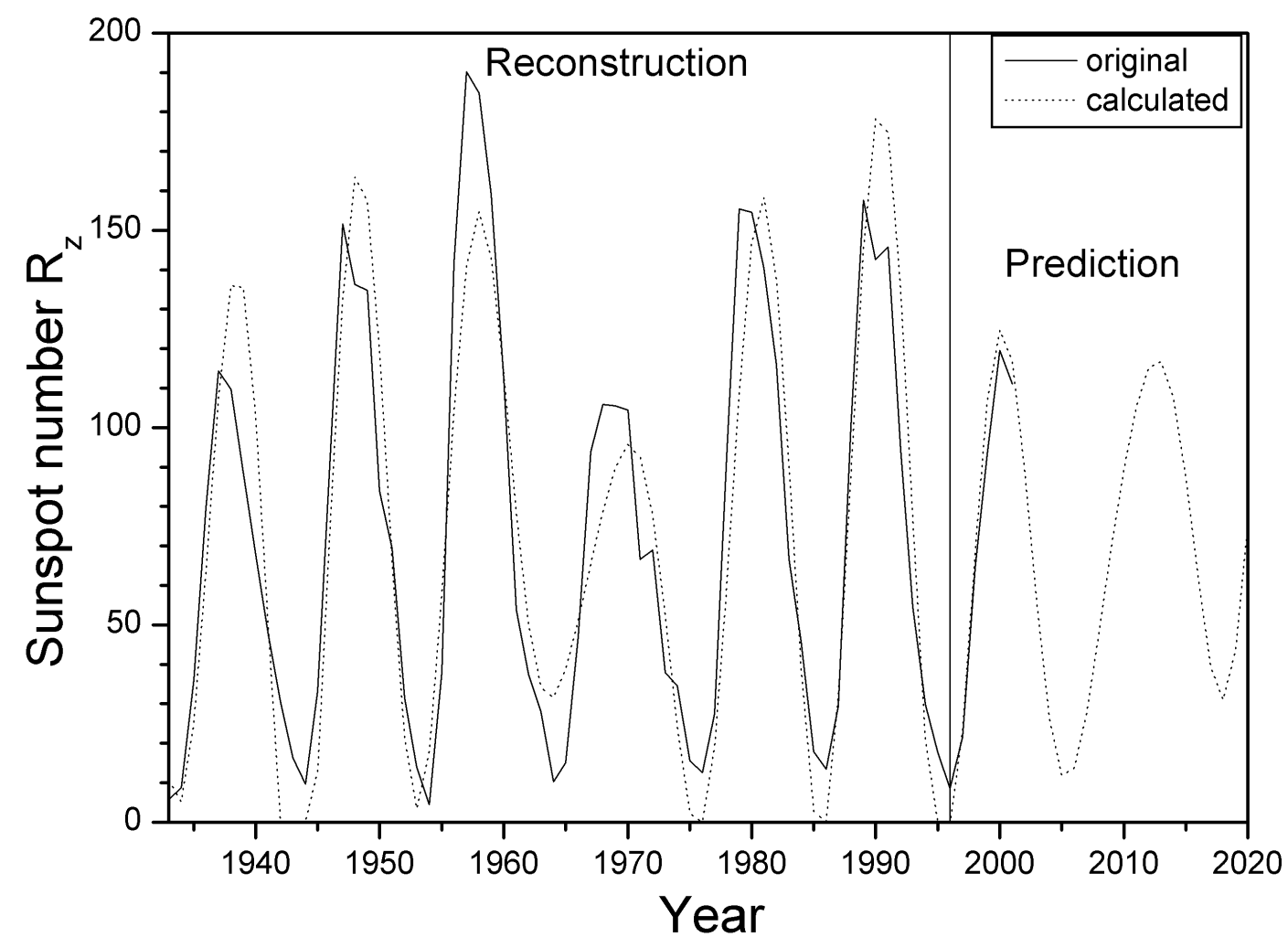

Fig. 2. Reconstructed/forecasted (dotted lines) and original (continuous line) sunspot number series. The continuous line in 1996 divides the reconstructed and predicted series. 
solar activity. Kane (1999) has shown that spectral characteristics are different for earlier (until 1914) and more recent sunspot data. In the present study the period 1933-1996 (solar cycle minima 17-22) was chosen to obtain the reconstruction, after testing shorter and longer periods. Data sample lengths shorter than the chosen one, for instance, 1951-1990, have an absence of long periods, resulting in short period sine waves in the reconstruction. Longer sample reconstructions, such as, for instance, 1901-1990, 1926-1980 and 1850-1990 underestimate several sunspot cycle maximum.

Using the ARIST method, the main periodicities of the 1933-1996 sunspot series were determined. The periods significant at the 95\% confidence level are: $8.5,10.5$ and 31.1 years. The 10.5 years is the solar cycle period, while the 8.5 years could be associated with it, because the solar cycle lengths are known to vary between 9-13 years (Hoyt and Schatten, 1997). The period of 31.1 years has been found elsewhere in sunspot number spectral analysis (Clúa de Gonzalez et al., 1993). A possibility is that this period of 31 years is the solar origin of the 35 years Brückner climatic periodicity (Raspopov et al., 2000).

Using Eq. (2) and the periodicities identified, a correlation analysis between the reconstructed and original data was performed, giving a high correlation coefficient of $\mathrm{r}=0.91$, implying a common variance $\left(\mathrm{r}^{2}\right)$ of about $80 \%$.

In Fig. 2 the reconstructed series (dotted line) and the original series are shown. The sunspot series was extrapolated until 2020 to predict solar cycles 23 and 24 . Some negative $\mathrm{R}_{z}$ values that were obtained by sine wave calculation were arbitrarily set to zero because they do not have physical significance. The correspondence is reasonable, especially in terms of sunspot cycle maximum amplitudes. Thus, in general, the reconstruction seems to be adequate to describe solar cycle behavior, including long-term periods, and it is able to fit adequately solar maximum values.

The error in the $R_{z}$ predicted value is calculated by the error theory as the square root of the sum of each periodicity square error:

$\sigma_{t}=\sqrt{\sigma_{1}^{2}+\sigma_{2}^{2}+\ldots+\sigma_{n}^{2}}$.

The amplitude and error of each periodicity are: $8.5 \mathrm{y}$ (29.5 \pm 8.7$) ; 10.5$ y $(65.6 \pm 4.2) ; 31.1$ y $(18.1 \mathrm{y} \pm 9.0)$. This gives an error for the reconstruction of 13.2. This estimated error is of the same order as obtained in other similar reconstructions, for instance, Kane (1999) found errors of around 10.0 .

It is seen in Fig. 2 that solar cycles 23 and 24 are predicted to be smaller than solar cycles 21 and 22. Predictions for solar cycle 21-23 were in general agreement with observed maximum peaks: \#21: 158 13.2 (1981) against 155.4 (1979) observed; \#22: $178 \pm 13.2$ (1990) against 157.6 (1989) observed; \#23: 125 13.2 (2000) against 119.6 (2000) observed. The prediction for solar maximum 24 is $117 \pm 13.2$ in year 2013, but the predicted value for 2012 is very close $(115 \pm 13.2)$. This could mean that solar cycle 24 would have a flat solar maximum, instead of a single peak as solar cycle 23.

Kane (2001) revised the performance of several predictions and, of the 20 predictions; only 8 were in interval $122 \pm 20$ (arbitrarily chosen by him). Table 1 shows various predictions found in the literature as compared to observed values along with the results of this study.

It is seen that the results of this work are in closer agreement with solar cycle 23 maximum amplitude than most of the methods, which have predicted higher solar activity. A panel convened by NASA and NOAA (Joselyn et al., 1997) reached a consensus of predicted sunspot maximum of $160 \pm 30$, which is much higher than the one actually observed in 2000.

Errors that have been observed in this prediction scheme seem to be due to the limitation of the method itself and also because part of the nature (nonlinear features) of the time series is not very well described by the model. It is important to comment that, although the solar cycle is asymmetric (e.g. fast rise and slow decline), the approximation of sine waves describes quite well the general behavior of solar activity. Also, the fact that by using different intervals the prediction could be different is a drawback of the method, but its accuracy in predicting solar maximum peak does not seem to be worse than that of other methods (Kane, 2002).

Wilson (1992) and Cliver et al. (1996) analyzed the peak patterns of $R_{z}$ since 1860 and they arrived at the conclusion that even cycles should have lower peaks than the preceding odd solar cycle. If this trend continues in the future, then solar cycle 24 would be lower than 23 , a result that is in agreement with the present work prediction.

\section{Conclusions}

Reconstruction and prediction of sunspot number series was done using an iterative regression analysis. Periodicities significant at the $95 \%$ confidence level have been selected in order to reconstruct the sunspot series during 1933-1996, with a correlation coefficient of $r=0.91$. Solar maximum peaks are adequately predicted, and prediction for solar cycle 23 were $125 \pm 13.2$ (against an observed peak of 119.6) and for solar cycle 24 of $115 \pm 13.2$ occurring in 2012 or $117 \pm 13.2$ occurring in 2013. Thus, the solar cycle 24 is predicted to be weaker than the last cycles.

Acknowledgements. We are grateful to the FAPESP "Fundação de Amparo a Pesquisa do Estado de Sao Paulo" for the financial support of our research (Contract 02/12723-2) and to the CNPq "Conselho nacional de Desenvolvimento Cientifíco e Tecnológico" for Research Fellowship (Contract PQ 300031/1994-7).

Topical Editor R. Forsyth thanks A. J. Conway and another referee for their help in evaluating this paper. 


\section{References}

Ahluwalla, H. S.: Solar Cycle 23 Prediction Update, Adv. Space Res., 26, 187, 2000.

Bounar, K. H., Cliver, E. W., and Boriakoff, V.: A prediction of the peak sunspot number for solar cycle 23, Solar Phys., 176, 211, 1997.

Cliver, E. W., Boriakoff, V., Bounar, K. H.: The 22-year cycle of geomagnetic and solar wind activity, J. Geophys. Res., 101, 27 091, 1996.

Clúa de Gonzalez, A. L., Gonzalez, W. D., Dutra, S. L. G., and Tsurutani, B. T.: Periodic variation in the geomagnetic activity: a study based on the $A_{p}$ Index, J. Geophys. Res., 98, 9215, 1993.

Conway, A. J.: Time series, neural networks and the future of the sun, New Astronomy Reviews, 42, 343-394, 1998.

Echer, E., Rigozo, N. R., Souza, M. P., Vieira, L. E. A., and Nordemann, D. J. R.: Reconstruction of the aa index on the basis of spectral characteristics, Geofísica Internacional, 43, 103, 2004.

Eddy, J. A.: The Maunder minimum, Science, 192, 1189, 1976.

Eddy, J. A.: The solar output and its variation: O. R. White, Colorado Associated University Press, Boulder, 1977.

Fyodorov, M. V., Klimenko, V. V., and Dovgalyuk, V. V.: Solar Phys., 165, 193, 1996.

Hathaway, D. H., Wilson, R. M., and Reichmann, E. J.: A synthesis of solar cycle prediction techniques, J. Geophys. Res. 104, $22375,1999$.

Herman, J. R. and Goldberg, R. A.: Sun, weather and climate, Scientific and Technical Information Branch, National Aeronautics and Space Administration, Washington D.C., 1978.

Hoyt, D. V. and Schatten, K. H.: The role of the sun in climate change, Oxford University Press, New York, 1997.

Hoyt, D. V. and Schatten, K. H.: Group sunspot numbers: A new solar activity reconstruction, Solar Phys., 179, 189, 1998a.

Hoyt, D. V. and Schatten, K. H.: Group sunspot numbers: A new solar activity reconstruction, Solar Phys., 181, 491, 1998b.

Jain, R.: Prediction of the amplitude of sunspot cycle 23, Solar Phys. 176, 431, 1997.

Joselyn, J. A., Anderson, J. B., Coffey, H., Harvey, K., Hathaway, D., Heckman, G., Hildner, E., Mende, W., Schatten, K., Thompson, R., Thompson, A. W. P., and White, O. R.: Panel achieves consensus prediction of solar cycle 23, EOS, 78, 205, 1997.

Kane, R. P.: Predicted intensity of the solar maximum, Nature, 274, 139, 1978

Kane, R. P.: A preliminary estimate of the size of the coming solar cycle 23, Geophys. Res. Lett., 24, 1899, 1997.

Kane, R. P.: Prediction of the sunspot maximum of solar cycle 23 by extrapolation of spectral components, Solar. Phys., 189, 217, 1999.

Kane, R. P.: Did predictions of the maximum sunspot number for solar cycle 23 come true?, Solar Phys., 202, 395, 2001.

Kane, R. P.: Prediction of solar activity: Role of long-term variations, J. Geophys. Res., 107, doi:10.1029/2001JA0700247, 2002.
Layden, A. C., Fox, P. A., Howard, J. M., Sarajedini, A., Schatten, K. H., and Sofia, S.: Dynamo-based scheme for forecasting the magnitude of solar activity cycles, Solar Phys., 132, 1, 1991.

Loskutov, A. Y., Istomin, I. A., Kothyarov, O. L., and Kuzanyan, K. M.: A study of the regularities in solar magnetic activity by singular spectral analysis, Astronomy Letters, 27, 745, 2001.

Li, Y.: Predictions of the features for sunspot cycle 23, Solar Phys., 170, 437, 1997.

Mendoza, B. and Ramírez, J.: A straightforward estimation of the maximum sunspot number for cycle 23, Ann. Geophys., 17, $17639,1999$.

Ohl, A. I.: Forecast of sunspot maximum number of cycle 20, Soln. Danie, 12, 84, 1966.

Ramesh, K. B.: Dependence of $\mathrm{SSN}_{M}$ on $\mathrm{SSN}_{m}-$ A reconsideration for predicting the amplitude of a sunspot cycle, Sol. Phys., 197, 421, 2000.

Raspopov, O. M., Shumilov, O. I., Kasatkina, E. A., Turunen, E., and Lindholm, M.: 35-year climatic Bruckner cycle-solar control of climate variability, Proc. 1stSolar and space weather euroconference, "The Solar Cycle and Terrestrial Climate", Santa Cruza de Tenerife, Tenerife, Spain, 2000.

Rigozo, N. R. and Nordemann, D. J. R.: Iterative regression analysis of periodicities in geophysical record time series, Rev. Bras. Geofis., 16, 149, 1998.

Rigozo, N. R., Echer, E., Vieira, L. E. A., and Nordemann, D. J. R.: Reconstruction of Wolf sunspot number on the basis of spectral characteristics and estimates of associated radio flux and solar wind parameters for the last millennium,Solar Phys., 203, 179, 2001.

Schatten, K. and Pesnell, W. D.: An early dynamo prediction: cycle 23 22, Geophys. Res. Lett., 20, 2275, 1993.

Schatten, K., Myers, B. J., and Sofia, S.: Solar activity forecast for solar cycle 23, Geophys. Res. Lett., 23, 605, 1996.

Schatten, K. and Sofia, S.: Bull. Am. Astron. Soc., 28, 1347, 1996.

Siscoe, G.: The space-weather enterprise: past, present and future, J. Atmos. Solar-Terr. Physics, 62, 1223, 2000.

Tompson, R. J.: The amplitude of solar cycle 23, Proc. Solar Terrestrial Predictions V, Proceedings of a workshop at Hitachi, Japan, January 1996.

Tsirulnik, L. B., Kuznetsova, T. V., and Oraevsky, N. V.: Forecasting the 23rd and 24th solar cycles on the basis of MGM spectrum, Adv. Space Res., 20, 2369, 1997.

Webb, D. F. and Howard, R. A.: The solar cycle variation of coronal mass ejections and the solar wind mass flux, J. Geophys. Res., 99, 4201, 1994.

Wilson, R. M.: An early estimate for the size of cycle 23, Sol. Phys., 140, 181, 1992.

Wolberg, J. R.: Prediction Analysis, Van Nostrand Company, Princeton, N. J., 291 pp, 1967. 